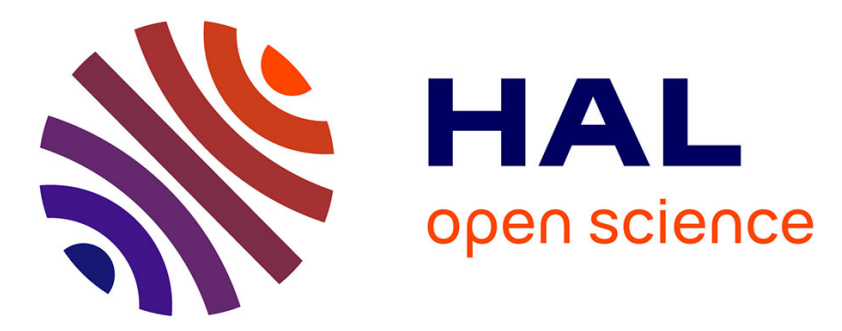

\title{
Numerical determination of the mean value of an elasticity tensor by integration over the rotation group with Haar measure
}

K. Atchonouglo, C. Vallé, Z.Q. Feng, J. Chaoui

\section{- To cite this version:}

K. Atchonouglo, C. Vallé, Z.Q. Feng, J. Chaoui. Numerical determination of the mean value of an elasticity tensor by integration over the rotation group with Haar measure. British Journal of Mathematics and Computer Science, 2015, 10 (4), 10.9734/BJMCS/2015/19480 . hal-02398146

\section{HAL Id: hal-02398146 \\ https://hal-univ-evry.archives-ouvertes.fr/hal-02398146}

Submitted on 4 Jun 2021

HAL is a multi-disciplinary open access archive for the deposit and dissemination of scientific research documents, whether they are published or not. The documents may come from teaching and research institutions in France or abroad, or from public or private research centers.
L'archive ouverte pluridisciplinaire HAL, est destinée au dépôt et à la diffusion de documents scientifiques de niveau recherche, publiés ou non, émanant des établissements d'enseignement et de recherche français ou étrangers, des laboratoires publics ou privés.

\section{(c)(1)}

Distributed under a Creative Commons Attribution| 4.0 International License 
British Journal of Mathematics \& Computer Science 10(4): 1-9, 2015, Article no.BJMCS.19480 ISSN : 2231-0851

SCIENCEDOMAIN international www.sciencedomain.org

Numerical Determination of the Mean Value of an Elasticity Tensor by Integration over the Rotation Group with Haar Measure

\author{
Kossi Atchonouglo ${ }^{1 *}$, Claude Vallée ${ }^{2}$, Zi-Qiang Feng ${ }^{3,4}$ \\ and Jamal Chaoufi ${ }^{5}$ \\ ${ }^{1}$ Département de Physique, Université de Lomé, Faculé des Sciences, \\ BP 1515 Lomé, Togo. \\ ${ }^{2}$ Institut P', Université de Poitiers, CNRS - ENSMA, UPR 3346, BP 30179, \\ 86962 Futuroscope - Chasseneuil Cedex, France. \\ ${ }^{3}$ School of Mechanics and Engineering, Southwest Jiaotong University, Chengdu, China. \\ ${ }^{4}$ Université d'Evry - Val d'Essonne - L.M.E., EA3332, 40 Rue du Pelvoux, 91020 Evry Cedex, \\ France. \\ ${ }^{5}$ Département de Physique, Université d'Agadir, Faculté des Sciences, Cité Dakhla, B.P. 8106, \\ 80000 Agadir, Morocco. \\ Article Information \\ DOI: 10.9734/BJMCS/2015/19480 \\ Editor(s): \\ (1) Nikolaos Dimitriou Bagis, Department of Informatics and Mathematics, A $\overline{\text { ristotelian }}$ \\ University of Thessaloniki, Greece. \\ Reviewers: \\ (1) G. Y. Sheu, Accounting and Information Systems, Chang-Jung Christian University, Tainan, \\ Taiwan. \\ (2) Manoel F Borges, Sao Paulo State University, Brazil. \\ (3) Grienggrai Rajchakit, Department of Mathematics, Mae jo University, Thailand. \\ Complete Peer review History: http://sciencedomain.org/review-history/10252
}

Original Research Article

Received: 11 June 2015

Accepted: 04 July 2015

Published: 19 July 2015

\begin{abstract}
Let $\mathbf{A}$ be a 3D symmetric elasticity tensor not necessary isotropic. If $\mu$ is an invariant measure on $S O(3)$, then $\mu$ is a convex combinaison of the Haar measure. The nearest isotropic elasticity tensor is obtained by integrating the tensor $\mathbf{A}$ on the rotation group $S O(3)$. For the numerical approach, we integrate the elasticity tensor on the unit ordinary ball $B(0,1)$.
\end{abstract}

Keywords: Elasticity tensor; Finite elements; identification. 2010 Mathematics Subject Classification: 53C25; 83C05; 57N16

*Corresponding author: E-mail: kossi.atchonouglo@gmail.com 


\section{Introduction}

In linear elasticity, the stress tensor and the strain tensor of a material are connected at a given temperature, by Hooke's law. Thus, each material is characterized by its elasticity tensor A, but this correspondence is not unique. Indeed, given the explicit component of $\mathbf{A}$ depends on the choice of an orthonormal basis in which are expressed tensor components. A change of orthonormal basis induces an action of the rotation group $S O(3)$ on the space of elasticity tensors $(\mathbb{E} l a)$. Therefore, from the viewpoint of linear elasticity, describing all materials is to describe the orbits of the action of the rotation group $S O(3)$ on $\mathbb{E} l a$.

Chandwick et al. [1] proved that there are eight classes which can be put into correspondence with the eight classical symmetry groups as described by Forte and Vianello [2]: triclinic, monoclinic, tetragonal, trigonal, orthotropic, transversely isotropic, cubic and isotropic materials. We are interested here on the isotropic elasticity tensors.

Let $\mathbf{A}_{0}$ be a raw elasticity tensor (because of the experimental errors) determined by any experimental method, e.g., the acoustical one presented by François et al. [3,4].

In the literature the least squares method is applied to research the nearest isotropic law. François et al. $[4,5]$ propose an intrinsic function that creates from the raw tensor $\mathbf{A}_{\mathbf{0}}$ and the arbitrary base $B$ a tensor $\mathbf{A}_{\mathbf{b}}$ which has the chosen symmetric group $G$. This function calculates the average of $\mathbf{A}_{\mathbf{0}}$ on its orbit according to $G_{B}$ related to the base $B$. The natural symmetry base $B_{s}$ is the one for which the relative discrepancy $D(B)$ between $\mathbf{A}_{\mathbf{b}}$ and $\mathbf{A}_{\mathbf{0}}$ can be called the distance from $\mathbf{A}_{\mathbf{0}}$ to $\mathbf{A}_{\mathbf{s}}$; in other words the distance to the symmetric group $G$.

This approach was also developped by M. Vianello in [6] where he minimizes the distance between the raw elasticity tensor measured and its invariants polynomial decomposition.

There are also some methods of parameterization of $S O(3)$ to determine the elasticity tensor.

A. Boná et al. [7] propose a parametrization of the twenty-one-dimensional space of elasticity tensors by eighteen parameters and three Euler angles in such a way that Euler angles determine the orientation of a natural coordinate system. Six of these parameters represent the rigidity moduli of the elasticity tensor, an-other twelve parameters determine the six orthonormal eigentensors with respect to the three orthonormal eigenvectors.

Sandra Forte et al. [8] propose a decomposition based on the Haar integral. Using the property of the left and right invariance of the Haar integral, they calcultate the restricted invariants on the space of elasticity tensors. The linear elasticity tensor is a linear combinaison of elements of the orbit centered at the origin.

The aim of this article is that we (effectively) calculated numerically the average on the group of $S O(3)$ by performing (by the parameterization of Olinde Rodrigues) the integration on a threedimensional unit ball (discretized by finite elements).

The validation example is a numerical simulation (noisy isotropic law) and not an experimental measurement of the elastic coefficients of a real material.

All vector spaces, matrices, ect., considered in this paper are real.

Latin indices vary in the set $\{1,2,3\}$, save when they are used for indexing sequences, and sommation convention with respect to repeated indices is systematically used in conjunction with this rule.

Spaces of functions, vector fields, and symmetric matrix fields, defined over an open subset are respectivelly denoted by italic capitals, boldface Romain, and spacial Roman capitals. 


\section{Numerical Integration on $S O(3)$}

\subsection{Hooke's Law}

Chandwick et al. [1] : in relation to some orthogonal basis, the components of the stress and strain tensors are $\sigma_{i j}$ and $\epsilon_{i j}$, respectively and Hooke's law takes the form $\sigma_{i j}=\mathbf{A}_{i j k l} \epsilon_{k l}$ where $\mathbf{A}_{i j k l}$ are the components of the fourth-order elasticity tensor $\mathbf{A}$. The components of $\mathbf{A}$ satisfy the symmetry relations $\mathbf{A}_{i j k l}=\mathbf{A}_{j i k l}=\mathbf{A}_{i j l k}=\mathbf{A}_{k l i j}$, due to the symmetry of the stress and strain tensors and the requierement that no net work be done by an elastic material in a closed loading cycle.

\subsection{Classical Determining of the Tensor A}

Let $\mathbf{A}$ be an elasticity tensor. We are looking for the nearest isotropic elasticity tensor.

Let us define the linar mapping : $\mathbf{H}(\epsilon)=\lambda \operatorname{tr}(\epsilon) \mathbf{I}+2 \mu \epsilon=\mathbf{A} \epsilon$. Hence, $\lambda$ and $\mu$ are the unkown parameters since the 21 coefficients of $\mathbf{A}$ are given.

For the classical method, the problem leads to minimize $\mathcal{P}(\lambda, \mu)=\frac{1}{2} \operatorname{tr}\left[(\mathbf{A}-\mathbf{H})^{2}\right]$. $\mathcal{P}$ is a second degree polynomial since $\mathbf{H}$ and $\mathbf{A}$ are explicited in Voigt normalized basis:

$$
\begin{aligned}
& \mathbf{E}_{1}=\left(\begin{array}{ccc}
1 & 0 & 0 \\
0 & 0 & 0 \\
0 & 0 & 0
\end{array}\right) ; \quad \mathbf{E}_{2}=\left(\begin{array}{ccc}
0 & 0 & 0 \\
0 & 1 & 0 \\
0 & 0 & 0
\end{array}\right) ; \quad \mathbf{E}_{3}=\left(\begin{array}{ccc}
0 & 0 & 0 \\
0 & 0 & 0 \\
0 & 0 & 1
\end{array}\right) ; \\
& \mathbf{E}_{4}=\frac{1}{\sqrt{2}}\left(\begin{array}{lll}
0 & 1 & 0 \\
1 & 0 & 0 \\
0 & 0 & 0
\end{array}\right) ; \quad \mathbf{E}_{5}=\frac{1}{\sqrt{2}}\left(\begin{array}{lll}
0 & 0 & 1 \\
0 & 0 & 0 \\
1 & 0 & 0
\end{array}\right) ; \quad \mathbf{E}_{6}=\frac{1}{\sqrt{2}}\left(\begin{array}{lll}
0 & 0 & 0 \\
0 & 0 & 1 \\
0 & 1 & 0
\end{array}\right)
\end{aligned}
$$

For the isotropic material, the elasticity tensor is written in Voigt basis:

$$
\mathbf{A}=\left(\begin{array}{ccccccc}
\lambda+2 \mu & \lambda & \lambda & 0 & 0 & 0 & \\
\lambda & \lambda+2 \mu & \lambda & 0 & 0 & & \\
\lambda & \lambda & \lambda+2 \mu & 0 & 0 & 0 & \\
0 & 0 & 0 & \mu & 0 & 0 & 0 \\
0 & 0 & 0 & \mu & 0 & & \\
0 & 0 & 0 & 0 & 0 & \mu &
\end{array}\right)
$$

One can explicit $\mathbf{H}$ and $\mathbf{A}$ on the basis constituted of five deviatorics and a spherical matrices:

$$
\mathbf{H}=\left(\begin{array}{cccc}
2 \mu & & (0) & \\
(0) & \ddots & & 0 \\
& 0^{T} & 2 \mu & \\
& & 3 \lambda+2 \mu
\end{array}\right) \quad \text { and } \mathbf{A}=\left(\begin{array}{cc}
\mathbf{S} & \mathbf{v} \\
\mathbf{v}^{T} & \alpha
\end{array}\right)
$$

where $\mathbf{S}$ is a $5 \times 5$ symmetric matrix, $\mathbf{v} \in \mathbb{R}^{5}$ and $\alpha \in \mathbb{R}$.

Let $\mathbf{I}_{5}$ be the $5 \times 5$ identity matrix, then the polynomial $\mathcal{P}(\lambda, \mu)$ is written

$$
\mathcal{P}(\lambda, \mu)=\frac{1}{2}[\alpha-(3 \lambda+2 \mu)]^{2}+10 \mu^{2}-2 \mu \operatorname{tr}\left[\left(\mathbf{S}-2 \mu \mathbf{I}_{5}\right)^{2}\right]+\mathbf{v}^{T} \mathbf{v} .
$$

For the classical approach, one has:

$$
3 \lambda+2 \mu=\alpha \text { and } 2 \mu=\frac{1}{5} \operatorname{tr} \mathbf{S} .
$$

This choice is justified by the fact that the elasticity tensor is diagonal in this basis and calculations are more simple.

Let us develop a new approach inspired from Sourriau [9]. 


\subsection{Integration on the Rotation Group $S O(3)$}

$S O(3)$ is a semisimple Lie group or unimodular group.

Let $\mathbf{R}$ be the $3 \times 3$ rotation matrix. We define the Haar positive left invariant measure $d \mu$. Since $S O(3)$ is unimodular group, this meausure is also right invariant (see [10]).

First, we will parametrize rotation matrices with Rodrigues coefficients.

\subsection{Rotation Parameterization}

Consider a rotation with the angle $\theta$ around the unit vector $\mathbf{u}$, the Gibbs formula is given by:

$$
\mathbf{R}=\cos (\theta) \mathbf{I}_{3}+\sin (\theta) j(\mathbf{u})+(1-\cos (\theta)) \mathbf{u} \otimes \mathbf{u}, \theta \in[0, \pi],
$$

where we define the matrix $j(\mathbf{u})=\left(\begin{array}{ccc}0 & -u_{3} & u_{2} \\ u_{3} & 0 & -u_{1} \\ -u_{2} & u_{1} & 0\end{array}\right)$.

The Rodrigues coefficients are:

$$
m_{0}=\cos \left(\frac{\theta}{2}\right) ; \quad m_{i}=\sin \left(\frac{\theta}{2}\right) u_{i}, i=1,2,3 .
$$

If we denote $\mathbf{m}=\left(m_{1}, m_{2}, m_{3}\right)^{T}$, we can rewrite the Gibbs formula:

$$
\mathbf{R}=\left(2 m_{0}^{2}-1\right) \mathbf{I}_{3}+2 m_{0} j(\mathbf{m})+2 \mathbf{m} \otimes \mathbf{m} .
$$

This expression is second degree respect to the parameters.

Since $0 \leq \theta \leq \pi$, the real $m_{0} \in[0,1]$. From $m_{0} \geq 0$ and $\left(m_{0}\right)^{2}+\left(m_{1}\right)^{2}+\left(m_{2}\right)^{2}+\left(m_{3}\right)^{2}=1$, one has: $m_{0}=\sqrt{1-\mathbf{m} . \mathbf{m}}$. Therefore the vector $\mathbf{m}$ is an element of the unit compact ball $B(0,1)$, and consequently $S O(3)$ is compact and we can parametrize $S O(3)$ by the Rodrigues parameters $m_{1}$, $m_{2}$ and $m_{3}[11,12]$.

Proposition 2.1. The unique Haar measure is

$$
d \mu=\frac{1}{\pi^{2}} \frac{d m_{1} d m_{2} d m_{3}}{m_{0}} .
$$

Comments Instead of integrating on $\mathrm{SO}(3)$ one integrates on the unit ordinary ball $\mathrm{B}(0,1)$ :

$$
\int_{S O(3)} f(\mathbf{R}) d \mu=\int_{B(0,1)} f(\mathbf{R}(\mathbf{m})) \frac{1}{\pi^{2}} \frac{d m_{1} d m_{2} d m_{3}}{\sqrt{1-\mathbf{m} \cdot \mathbf{m}}} .
$$

Let us verify that $\int_{B(0,1)} \frac{d m_{1} d m_{2} d m_{3}}{m_{0}}=\pi^{2}$.

$$
\begin{aligned}
\int_{B(0,1)} \frac{d m_{1} d m_{2} d m_{3}}{\sqrt{1-\mathbf{m} \cdot \mathbf{m}}} & =\int_{0}^{1} \frac{S(r)}{\sqrt{1-r^{2}}} d r S(r) \text { is the sphere surface } \\
& =4 \pi \int_{0}^{1} \frac{r^{2} d r}{\sqrt{1-r^{2}}}=\pi \int_{0}^{\pi}(1-\cos \theta) d \theta=\pi^{2}
\end{aligned}
$$

Proof. of the proposition : We seek the Haar measure in density measure form

$$
d \mu=\rho(\mathbf{m}) d m_{1} d m_{2} d m_{3} .
$$


Let us prove that the left-invariance imposes that

$$
\rho(\mathbf{m})=\frac{C}{\sqrt{1-\left(m_{1}\right)^{2}-\left(m_{2}\right)^{2}-\left(m_{3}\right)^{2}}}
$$

where $C$ is a constant.

One can verify that the right-invariance leads to the same conclusion.

The definition $\mathbf{R}^{\prime}=\Omega \mathbf{R}, \Omega \in S O(3)$ leads to [12]

$$
\left\{\begin{array}{l}
n_{0}^{\prime}=m_{0} n_{0}-\mathbf{m} . \mathbf{n} \\
\mathbf{m}^{\prime}=m_{0} \mathbf{n}+\mathbf{m} \wedge \mathbf{n} .
\end{array}\right.
$$

The invarianbility of the Haar measure $d \mu=\rho(\mathbf{m}) d \mathbf{m}$ is equivalent to the condition

$$
\rho\left(\mathbf{m}^{\prime}\right) \mathbf{d} \mathbf{m}^{\prime}=\rho(\mathbf{m}) \mathbf{d} \mathbf{m} .
$$

Let the matrix $\mathbf{J}$ be the jacobean of the mapping which transforms $\mathbf{m}$ to $\mathbf{m}^{\prime}$. Haar measure imposes that for all $\mathbf{n}, \rho\left(\mathbf{m}^{\prime}\right) \operatorname{det} \mathbf{J}=\rho(\mathbf{m})$.

Now, let us calculate the jacobean $\mathbf{J}$.

First, we verify that $\left(m_{0}^{\prime}\right)^{2}+\mathbf{m}^{\prime} \cdot \mathbf{m}=1$.

Indeed,

$$
\begin{aligned}
\left(\begin{array}{l}
m_{0}^{\prime} \\
\mathbf{m}^{\prime}
\end{array}\right) & =\left(\begin{array}{cc}
n_{0} & -\mathbf{n}^{T} \\
\mathbf{n} & n_{0} \mathbf{I}_{3}-j(\mathbf{n})
\end{array}\right)\left(\begin{array}{c}
m_{0} \\
\mathbf{m}
\end{array}\right) \text { where } j(\mathbf{n})=\left(\begin{array}{ccc}
0 & -n_{3} & n_{2} \\
n_{3} & 0 & -n_{1} \\
-n_{2} & n_{1} & 0
\end{array}\right) \\
& =n_{0} \mathbf{I}_{4}+n_{1}\left(\begin{array}{cccc}
0 & -1 & 0 & 0 \\
1 & 0 & 0 & 0 \\
0 & 0 & 0 & 1 \\
0 & 0 & -1 & 0
\end{array}\right)+n_{2}\left(\begin{array}{cccc}
0 & 0 & -1 & 0 \\
0 & 0 & 0 & -1 \\
1 & 0 & 0 & 0 \\
0 & 1 & 0 & 0
\end{array}\right)+n_{3}\left(\begin{array}{cccc}
0 & 0 & 0 & -1 \\
0 & 0 & 1 & 0 \\
0 & -1 & 0 & 0 \\
1 & 0 & 0 & 0
\end{array}\right)
\end{aligned}
$$

The product

$$
\left(\begin{array}{cc}
n_{0} & -\mathbf{n}^{T} \\
-\mathbf{n} & n_{0} \mathbf{I}_{3}+j(\mathbf{n})
\end{array}\right)\left(\begin{array}{cc}
n_{0} & -\mathbf{n}^{T} \\
\mathbf{n} & n_{0} \mathbf{I}_{3}-j(\mathbf{n})
\end{array}\right)
$$

is the $4 \times 4$ identity matrix.

Secondly, to calculate the jacobean one can write

$$
\left(\begin{array}{l}
\delta m_{0}^{\prime} \\
\delta \mathbf{m}^{\prime}
\end{array}\right)=\left(\begin{array}{cc}
n_{0} & -\mathbf{n}^{T} \\
\mathbf{n} & n_{0} \mathbf{I}_{3}-j(\mathbf{n})
\end{array}\right)\left(\begin{array}{l}
\delta m_{0}^{\prime} \\
\delta \mathbf{m}^{\prime}
\end{array}\right)
$$

Since one has $\delta m_{0}=-\frac{1}{m_{0}} \mathbf{m} . \delta \mathbf{m}$ and $\delta m_{0}=\frac{1}{m_{0}^{\prime}} \mathbf{m}^{\prime} . \delta \mathbf{m}^{\prime}$, therefore

$$
\left(\begin{array}{l}
\delta m_{0}^{\prime} \\
\delta \mathbf{m}^{\prime}
\end{array}\right)=\left(\begin{array}{c}
-\frac{\mathbf{m}^{T}}{m_{0}} \\
\mathbf{I}_{3}
\end{array}\right) \delta \mathbf{m}
$$

Finaly, the jacobean matrix is the $3 \times 3$ matrix

$$
\mathbf{J}=\left(\begin{array}{ll}
0 & \\
0 & \mathbf{I}_{3} \\
0 &
\end{array}\right)\left(\begin{array}{cc}
n_{0} & -\mathbf{n}^{T} \\
\mathbf{n} & n_{0} \mathbf{I}_{3}-j(\mathbf{n})
\end{array}\right)\left(\begin{array}{c}
-\frac{\mathbf{m}^{T}}{m_{0}} \\
\mathbf{I}_{3}
\end{array}\right) .
$$

Once we calculate $\mathbf{J}$, one realizes, due to the compatibility conditions, that the condition (2.2) is only possible if $\rho(\mathbf{m})=\frac{\text { constant }}{m_{0}}$. 


\subsection{Rotation Group Action on the Elasticity Tensor}

Let $\mathbb{E} l a$ be a $3 \mathrm{D}$ strain space. This space is Euclidean space if we consider the scalar product:

$$
\begin{aligned}
E \times E & \longrightarrow \mathbb{R} \\
\left(\epsilon, \epsilon^{\prime}\right) & \longmapsto \operatorname{tr}\left(\epsilon \epsilon^{\prime}\right)
\end{aligned}
$$

An elasticity tensor is a self-adjoint linear mapping.

We define a $S O(3)$ group action on the six-dimensional vectorial space of elasticity tensors by:

$$
\forall \epsilon \in E, \forall \mathbf{R} \in S O(3): \mathbf{A}_{R}(\epsilon)=\mathbf{R A}\left(\mathbf{R}^{-1} \epsilon \mathbf{R}\right) \mathbf{R}^{-1} .
$$

We denote by $\mathbf{R}(\mathbf{A})=\mathbf{A}_{R}$ this action. For two rotations $\mathbf{R}_{1}$ and $\mathbf{R}_{2}$ we can verify that

$$
\left(\mathbf{R}_{1} \mathbf{R}_{2}\right)(\mathbf{A})(\epsilon)=\mathbf{R}_{1} \mathbf{R}_{2} \mathbf{A}\left(\mathbf{R}_{2}^{-1} \mathbf{R}_{1}^{-1} \epsilon \mathbf{R}_{1} \mathbf{R}_{2}\right) \mathbf{R}_{2}^{-1} \mathbf{R}_{1}^{-1}=\mathbf{R}_{1}\left(\mathbf{R}_{2}(\mathbf{A})\right)(\epsilon)
$$

Then, for the elasticity tensors, the action satisfies the group action law

$$
\left(\mathbf{R}_{1} \mathbf{R}_{2}\right)(\mathbf{A})=\mathbf{R}_{1}\left(\mathbf{R}_{2}(\mathbf{A})\right) .
$$

if $\mathbf{A}$ is isotropic, therefore $\mathbf{R}(\mathbf{A})=\mathbf{A}$ for all $\mathbf{R}$. If due to steps approximation, the tensor $\mathbf{A}$ is not isotropic, one can take the combination of $\mathbf{R}$ on the $S O(3)$ group

$$
\mathbf{H}=\int_{S O(3)} \mathbf{R}(\mathbf{A}) d \mu .
$$

Proposition 2.2. This combination of $\mathbf{R}$ on the $S O(3)$ group is isotropic.

Remark 2.1. For the numerical implementation, we use the Rodrigues coefficients to integrate:

$$
\mathbf{H}=\frac{1}{\pi^{2}} \int_{B(0,1)} \mathbf{R}(\mathbf{m}) \mathbf{A} \frac{d \mathbf{m}}{m_{0}} .
$$

We calculate $\mathbf{H}$ by the finite element method.

Proposition 2.3. $\int_{S O(3)} \mathbf{R}(\boldsymbol{A}) d \mu$ is the solution of the minimization problem of the classical method.

Proof. this proposition is due from the fact that $S O(3)$ preserves the lengths.

Let us prove that

$$
3 \mu^{\prime}+2 \lambda^{\prime}=\alpha \quad \text { and } \quad 2 \mu^{\prime}=\frac{1}{5} \operatorname{tr} \mathbf{S}
$$

For the first assertion, one has $\mathbf{R}(\mathbf{A})=\mathbf{R} \mathbf{A}\left(\mathbf{R}^{-1} \epsilon \mathbf{R}\right) \mathbf{R}^{-1}$.

Let $\epsilon=\mathbf{I}_{3}$, we obtain $\mathbf{R}(\mathbf{A})=\mathbf{R A}\left(\mathbf{I}_{3}\right) \mathbf{R}^{-1}$.

We can rewrite $\mathbf{A}=\alpha \frac{\mathbf{I}_{3}}{\sqrt{3}} \otimes \frac{\mathbf{I}_{3}}{\sqrt{3}} ;$ therefore $\mathbf{A I}_{3}=\alpha \frac{\mathbf{I}_{3}}{3}$ and $\operatorname{tr}\left[\mathbf{I}_{3}\left(\mathbf{R}(\mathbf{A}) \mathbf{I}_{3}\right)\right]=\operatorname{tr}\left[\mathbf{I}_{3} \mathbf{A}\left(\mathbf{I}_{3}\right)\right]=\alpha$.

Furthermore, from the formula $\mathbf{H}(\mathbf{A})=\frac{1}{\pi^{2}} \int_{B(0,1)} \mathbf{R}(\mathbf{m}) \mathbf{A} \frac{d \mathbf{m}}{m_{0}}$, we conclude

$$
\operatorname{tr}\left[\mathbf{I}_{3} \mathbf{H}\left(\mathbf{I}_{3}\right)\right]=3 \lambda^{\prime}+2 \mu^{\prime}=\frac{1}{\pi^{2}} \alpha \int_{B(0,1)} \frac{d \mathbf{m}}{m_{0}}=\alpha .
$$

For the second one, we rewrite

$$
\mathbf{H}(\mathbf{A})-\alpha \frac{\mathbf{I}_{3}}{\sqrt{3}} \otimes \frac{\mathbf{I}_{3}}{\sqrt{3}}=\frac{1}{\pi^{2}} \int_{B(0,1)} \mathbf{R}(\mathbf{m})\left[\mathbf{A}-\alpha \frac{\mathbf{I}_{3}}{\sqrt{3}} \otimes \frac{\mathbf{I}_{3}}{\sqrt{3}}\right] \frac{d \mathbf{m}}{m_{0}}
$$

Let us use this lemma: 
Lemma 2.1. The group action conserves the trace.

So, if we take the trace of both members, we get

$$
10 \mu^{\prime}=\frac{1}{\pi^{2}} \operatorname{tr} \mathbf{S} \int_{B(0,1)} \frac{d \mathbf{m}}{m_{0}}=\operatorname{tr} \mathbf{S} .
$$

Proof. of the lemma : we must prove that $\mathbf{R}(\mathbf{A})$ and $\mathbf{A}$ have the same trace.

One can take $\mathbf{R}(\mathbf{A})=e^{-\theta \mathbf{M}} \mathbf{A} e^{\theta \mathbf{M}}$ with $\mathbf{M} \epsilon=\epsilon j(\alpha)-j(\alpha) \epsilon$.

Then,

$$
\operatorname{tr} \mathbf{H}=\int_{S O(3)} \operatorname{tr}(\mathbf{A}) d \mu=\operatorname{tr} \mathbf{A} \int_{S O(3)} d \mu=\operatorname{tr} \mathbf{A} .
$$

\section{Numerical Example}

Instead of analytically integrating Eq. 2.3, a numerical approach is applied. The unit ordinary ball $B(0,1)$ is discretized into 270424 tetrahedral elements, as shown in Figure (1). Initial values

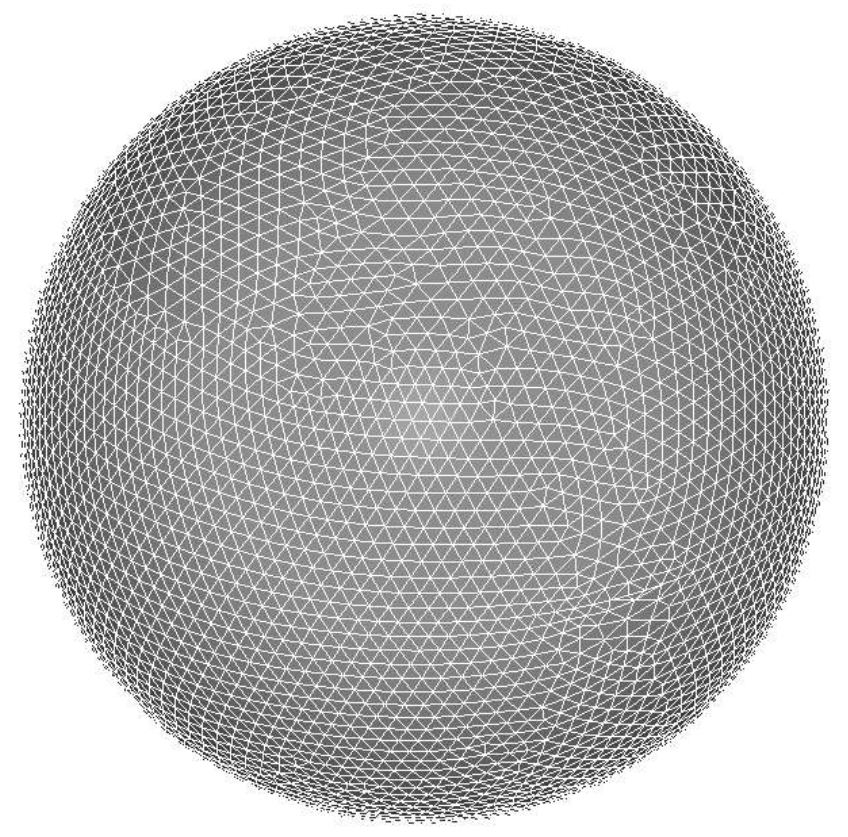

Figure 1: Discretized ball with 270424 elements

of material constants are chosen as: $\lambda=50 \mathrm{MPa}, \mu=10 \mathrm{MPa}$. A perturbation is applied to the elasticity tensor A by

$$
A_{i j k l}=A_{i j k l}^{0}+0.01[1+(i-1)(j-1)-0.32(k-1)(l-1)]
$$


For numerical purpose, it is more convenient to write the elasticity tensor in a symmetric matrix form based on Voigt's representation:

$$
[\mathbf{A}]_{o}=\left[\begin{array}{llllll}
70.01 & 50.0068 & 49.9972 & 0.01 & 0.0036 & 0.01 \\
& 70.0168 & 50.0072 & 0.02 & 0.0136 & 0.02 \\
& & 70.0372 & 0.05 & 0.0436 & 0.05 \\
& & & 10.01 & 0.0036 & 0.01 \\
& & & & 10.0236 & 0.0036 \\
& & & & & 10.01
\end{array}\right]
$$

After integration, we obtain new material constants as: $\lambda=46.9757 \mathrm{MPa}, \mu=9.4004 \mathrm{MPa}$. The elasticity matrix becomes:

$$
[\mathbf{A}]_{n}=\left[\begin{array}{llllll}
65.7766 & 46.9757 & 46.9758 & -0.000482 & -0.000257 & -0.000589 \\
& 65.7765 & 46.9757 & -0.000264 & -0.000381 & -0.000297 \\
& & 65.7762 & -0.000223 & -0.000085 & -0.000118 \\
& & & 9.40039 & -0.000364 & -0.000146 \\
& & & & 9.40026 & -0.000306
\end{array}\right]
$$

We remark that the two coefficients of Lamé are determined with good accuracy: the first coefficient $\lambda$ is determined with $6 \%$ accuracy, and the second $\mu$ is determined with $3 \%$ accuracy.

\section{Conclusion}

Based on the Souriau variational approach, we established that the nearest isotropic elasticity tensor of a measured elasticity tensor can be determined by integration on the rotation group $S O(3)$. The rotation matrices parametrization within Rodrigues coefficients leads to integrate on the unit ordinary ball $B(0,1)$. The unit ball is discretized by the finite element approach. The numerical results confirm the approach developped. The accuracies obtained on the Lamé's coefficients are very good.

In this work, the elasticity tensor was identified. We propose in the future to extend this approach to other symmetry group and this work is being undertaken.

\section{Competing Interests}

The authors declare that no competing interests exist.

\section{References}

[1] Chadwick P, Vianello M, Cowin SC. A new proof that the number of linear elastic symmetries is eight. Journal of the Mechanics and Physics of Solids. 2001;49(11):2471-2492.

[2] Forte S, Vianello M. Symmetry classes and harmonic decomposition for photoelasticity tensors. Internat. J. Engrg. Sci. 1997;35(14):1317-1326.

[3] François M, Geymonat G, Berthaud Y. Determination of the symmetries of an experimentally determined stiffness tensor; Application to acoustic measurements. Int. J. Solids Structures. 1998;(35):4091-4106.

[4] François M. Vers une mesure non destructive de la qualité des bois de lutherie. Revue des composites et des matériaux avancés. 2000;10(3):261-279. 
[5] François M, Berthaud Y, Geymonat G. Une nouvelle analyse des symétries d'un matériau anisotrope. Exemple d'utilisation à partir de mesures ultrasonores. Cr. Acad. Sci. IIb. 1996;322:87-94.

[6] Maurizio V. An Integrity basis for plane elasticity tensors. Archives of Mechanics. 1997;49(1):197-208.

[7] Bóna A, Bucataru I, Slawinski MA. Space of $S O(3)$-orbits of elasticity tensors. Archives of Mechanics. 2008;60(2):123-138.

[8] Sandra forte and maurizio vianello. Functional bases for transversely isotropic and transversely hemitropic invariants of elasticity tensors. The Quarterly Journal of Mechanics and Applied Mathematics. 1998;51(4):543-552.

[9] Souriau JM. Calcul linéaire. PUF, Paris; 1965.

[10] Wendel JG. Haar measure and the semigroup of measures on a compact group. Proceedings of the American Mathematical Society. 1954;5(6):923-929.

[11] Bouby C, Fortuné D, Pietraszkiewicz W, Vallée C. Direct determination of the rotation in the polar decomposition of the deformation gradient by maximizing a Rayleigh quotient. ZAMM - Journal of Applied Mathematics and Mechanics. 2005;85(3):155-16.

[12] Stuelpnagel J. On the Parametrization of the three-dimensional rotation group. SIAM Rev. 1964;6(4):422-430.

(C)2015 Atchonouglo et al.; This is an Open Access article distributed under the terms of the Creative Commons Attribution License http://creativecommons.org/licenses/by/4.0, which permits unrestricted use, distribution, and reproduction in any medium, provided the original work is properly cited.

Peer-review history:

The peer review history for this paper can be accessed here (Please copy paste the total link in your browser address bar)

http://sciencedomain.org/review-history/10252 